\title{
INTEGRACIÓN DE INFORMACIÓN AMAZÓNICA: SISTEMA DE INFORMACIÓN DE LA DIVERSIDAD BIOLÓGICA Y AMBIENTAL DE LA AMAZONÍA PERUANA
}

\author{
Víctor Miyakawa Solís ${ }^{1}$, Risto Kalliola ${ }^{2}$ y José Luis Mena Álvarez ${ }^{3}$
}

\begin{abstract}
RESUMEN
Como cualquier otra información, los datos sobre diversidad biológica y ambiental necesitan ser evaluados y clasificados cuidadosamente, para hacer útiles y compatibles a las herramientas de las tecnologías de información modernas. Los datos biológicos son muy variados, abarcando desde colecciones de especimenes biológicos en museos de historia natural, artículos científicos y libros publicados en diversos países y lenguajes, hasta investigadores individuales con especialización en una cierta área. Debido a tal heterogeneidad de las fuentes y la enorme cantidad de información, se considera que la implementación de un sistema de información debe seguir una pauta compartida, no funcionando solamente dentro de un único lugar o institución. La realización de este objetivo es muy posible gracias al uso de modernos equipos de computación y al establecimiento de una red de información. Este artículo presenta algunos lineamientos relativos al Sistema de Información de la Diversidad Biológica y Ambiental de la Amazonía Peruana - SIAMAZONIA, cuya arquitectura se basa en el establecimiento de nodos, los cuales representan a diversas instituciones que poseen información valiosa sobre diversidad biológica de la región amazónica peruana. Debido a su naturaleza versátil, los nodos se clasifican en tres tipos: un nodo facilitador, varios nodos principales, y nodos adicionales. SIAMAZONIA se desarrolla como una herramienta para facilitar la producción, intercambio y distribución de información sobre diversidad biológica y ambiental en Amazonía; así mismo se integra con el Mecanismo de Facilitación (Clearing House Mechanism) del Convenio sobre la Diversidad Biológica, y responde también a los requerimientos de desarrollo bajo el contexto del GBIF (Global Biodiversity Information Facility), donde el Perú ha comprometido su participación como país observador.
\end{abstract}

Palabras clave: Datos, diversidad biológica, información, informática, nodos, red de información, Sistema de Información de la Diversidad Biológica y Ambiental de la Amazonía Peruana - SIAMAZONIA, Web.

\footnotetext{
ABSTRACT

As with any kind of information, biodiversity and environmental data must be assessed and classified carefully in order to make it useful and compatible with the tools of modern information technologies. The information sources for biological data are highly variable, ranging from collections of biological specimens in natural history museums, to scientific articles and books published in different countries and languages, even individual researchers with specialization in some particular research area. Due to the heterogeneity of information sources and the overwhelming amount of information, it is thought that the implementation of an information system should be based on the concept of shared network, rather than operating within a single location or institute. This objective can be achieved through the use of modern computer equipment and the establishment of an information network. This article presents the Peruvian Amazon Biodiversity and Environmental Information System (SIAMAZONIA), which is based on the establishment of nodes that represent different institutions,

1 Proyecto Diversidad Biológica de la Amazonía Peruana - BIODAMAZ, Perú - Finlandia. Av. Abelardo Quiñones km 2.5, Iquitos, Perú. Correo electrónico: biodamaz@iiap.org.pe / Centro de Información de la Amazonía Peruana - CIAP, Instituto de Investigaciones de la Amazonía Peruana - IIAP. Av. Abelardo Quiñones km 2.5, Iquitos, Perú.

2 Proyecto Diversidad Biológica de la Amazonía Peruana - BIODAMAZ, Perú - Finlandia. Av. Abelardo Quiñones km 2.5, Iquitos, Perú / Departamento de Geografía, Universidad de Turku, 20014 Turku, Finlandia. Correo electrónico: biodamaz@iiap.org.pe

3 Proyecto Diversidad Biológica de la Amazonía Peruana - BIODAMAZ, Perú - Finlandia. Av. Abelardo Quiñones km 2.5, Iquitos, Perú. Correo electrónico: biodamaz@iiap.org.pe
} 
each of which possesses valuable information on biological diversity in the Peruvian Amazon region. Due to their versatile nature, the nodes are classified into three types: the facilitating node, several principal nodes, and additional nodes. SIAMAZONIA is developed as a tool to help the production, exchange and distribution of information on biological and environmental diversity in the Amazon, and also includes the Clearing House Mechanism, established by the Convention on Biological Diversity; it is also compatible with the development requirements of the Global Biodiversity Information Facility (GBIF), to which Peru has committed itself to participating as an observer country.

Key words: Biological diversity, data, informatics, information, information network, nodes, Peruvian Amazonian Biodiversity and Environmental Information System (SIAMAZONIA), Web.

\section{INTRODUCCIÓN}

La diversidad biológica es descrita comúnmente como la riqueza de genes, especies y ecosistemas. En todos sus niveles comprende una gran complejidad, especialmente en los bosques húmedos tropicales como los de la Amazonía. Este invalorable recurso está actualmente amenazado por las distintas actividades humanas, tales como deforestación y mal uso de los recursos naturales (Dourojeanni, 1990).

Para facilitar pautas más sostenibles del desarrollo, la recientemente publicada Estrategia Regional de la Diversidad Biológica Amazónica - ERDBA, precisa, entre otros aspectos, la necesidad de ampliar los conocimientos sobre los patrones de diversidad biológica y los procesos ecológicos, biológicos y ambientales que los sustentan, para formar bases para el desarrollo sostenible (BIODAMAZ, 2001a). En la Amazonía del Perú se conoce que los tipos de ecosistemas y su biogeografía implícita son el resultado de las diversas influencias de los factores ambientales y procesos históricos que caracterizan esta región (por ejemplo Kalliola et al., 1993). Dado que el conocimiento actual sobre esta diversidad y sus componentes aún es muy deficiente, éste no facilita la toma de decisiones fijas sobre la conservación y uso de estos ecosistemas.

Es importante precisar que la información que puede ser relevante en este sentido es muy compleja en su naturaleza (Tabla 1). Se considera que las colecciones de plantas en los herbarios y de animales en los museos zoológicos son registros muy concretos y evaluables. También los artículos y libros publicados sobre la diversidad biológica amazónica son fuentes importantes, así como también la información sobre las bibliotecas que mantienen estas publicaciones y registros sobre ellas. Aun más, los especialistas de diversos campos de flora y fauna, y hasta geografía, geología y climatología, pueden considerarse como pilares importantes de la información. Estos ejemplos confirman que estamos tratando un tema que es muy versátil en su naturaleza.

Tabla 1. Fuentes de información relevante sobre la diversidad biológica y ambiental amazónica.

\begin{tabular}{l|l}
\hline Tipo de información & Fuentes de conocimiento \\
\hline Colecciones biológicos & $\begin{array}{l}\text { Museos de Historia Natural, Herbarios, colecciones } \\
\text { biológicas personales }\end{array}$ \\
Expertos de investigación, conocimiento científico & Universidades, centros de investigación \\
Mapas e imágenes de percepción remota & Organizaciones públicas, organizaciones no gubernamentales \\
Otro conocimiento y expertos particulares & Organizaciones públicas, organizaciones no gubernamentales \\
Literatura & Bibliotecas publicas, institucionales y particulares; Internet \\
Bases de datos de estudios de campo & Cada uno de los antes mencionados \\
\hline
\end{tabular}


Al desarrollar estrategias de manejo de información sobre diversidad biológica es esencial considerar quiénes son los usuarios proyectados para este tipo de información, teniendo en cuenta que el interés sobre la diversidad biológica generalmente depende de motivaciones específicas. Por ejemplo, un investigador de monos puede planificar un estudio de campo por el río Tapiche; sería muy útil para él tener acceso a las imágenes de satélite de esta zona para ubicar y clasificar los diferentes tipos de bosques en la región. Adicionalmente, sería importante para este investigador consultar todas las publicaciones producidas de esta zona, para un mejor entendimiento de los lugares a visitar. En otro ejemplo, un planificador está comparando el impacto ambiental de dos rutas alternativas para una nueva carretera, para lo cual sería útil tener acceso a todo el conocimiento existente de los ecosistemas en ambas áreas, y no sólo realizar consultas a expertos que tienen experiencia de trabajo en estas zonas. Un sistema bien organizado de información sobre la diversidad biológica podría ayudar en ambas situaciones en una manera clara, precisa y rápida.

En el Convenio sobre la Diversidad Biológica (CDB), firmado en Río de Janeiro, en 1992, se define un mecanismo llamado "clearing house mechanism" (CHM, mecanismo de facilitación), designado para facilitar el intercambio de información sobre esta tema. Según este mecanismo, los poseedores de información, como científicos y otros investigadores, pueden presentar sus conocimientos, y aquéllos que necesitan esta información pueden consultar a expertos y sus conocimientos. Bien arreglado y en funcionamiento, y teniendo como plataforma el Internet, un sistema CHM puede formar una base importante para la definición de políticas adecuadas, de manera que faciliten el desarrollo sostenible. En el Perú, la responsabilidad del CHM nacional está a cargo del Consejo Nacional del Ambiente (CONAM), cuyo sitio Web puede ser encontrado en: http://www.conam.gob.pe/ chm. Asimismo, es importante reconocer la decisión del Perú (mayo 2001), para participar como país observador en el GBIF (Global Biodiversity Information Facility; Saarenmaa, 1998), que es una iniciativa internacional sumamente importante para manejar e intercambiar información sobre la diversidad biológica.

Por ser el Perú un país excepcionalmente diverso, se ha visto apropiado contar con esfuerzos regionales para sustentar el desarrollo de mecanismos más precisos sobre la diversidad biológica (BIODAMAZ 2001a; ENDB 2001). Se decidió desarrollar un sistema de información regional en la Amazonía peruana, siendo el Proyecto Diversidad Biológica de la Amazonía Peruana, Perú - Finlandia (BIODAMAZ) y el Instituto de Investigaciones de la Amazonía Peruana (IIAP) responsables del diseño y puesta en marcha de tal sistema. El presente artículo tiene sus raíces en dicho proceso, es decir, la creación del Sistema de Información de la Diversidad Biológica y Ambiental de la Amazonía Peruana, SIAMAZONIA. El sistema fue puesto en marcha en noviembre del 2001, y su sitio Web se encuentra en la dirección http://www.siamazonia.org.pe/.

El objetivo del presente artículo es identificar teórica y tecnológicamente las necesidades y posibilidades en los campos de informática, biología y biogeografía, los cuales forman la base para el desarrollo de ideas sobre el sistema amazónico. Señalamos que los últimos avances en la informática proveen poderosas herramientas para el análisis y manejo de información sobre los complejos procesos que involucran a la diversidad biológica. Si la información sobre ella es aún complicada y se encuentra dispersa, las redes y su utilización, complementadas con reglas y normativas compartidas, hacen posible la creación de un conjunto que reconoce la autoridad de cada información, supera limitaciones geográficas, y puede servir a sus usuarios las 24 horas del día.

\section{JERARQUÍA DE INFORMACIÓN}

Para ilustrar el flujo de información en la sociedad, es importante reconocer que existen diferentes niveles de la misma, desde los datos de observaciones originales hasta el entendimiento de los fenómenos por parte de teorías científicas y sabiduría (Figura 1). De acuerdo con esta visión, la información es un insumo estratégico y de apoyo para la adopción de decisiones que contribuyan al desarrollo integral de la sociedad. Fundamentalmente, los mismos tipos de requerimientos caracterizan a casi cualquier país y región del mundo (por ejemplo, Arriaga y Careaga, 1999; Laihonen et al., 2002).

4 Proyecto Diversidad Biológica de la Amazonía Peruana (BIODAMAZ) es un convenio entre los gobiernos del Perú y de Finlandia ejecutado conjuntamente por el Instituto de Investigaciones de la Amazonía Peruana (IIAP) y por el consorcio finlandés formado por la empresa de consultorías ambientales, Biota BD Oy, y por la Universidad de Turku. 


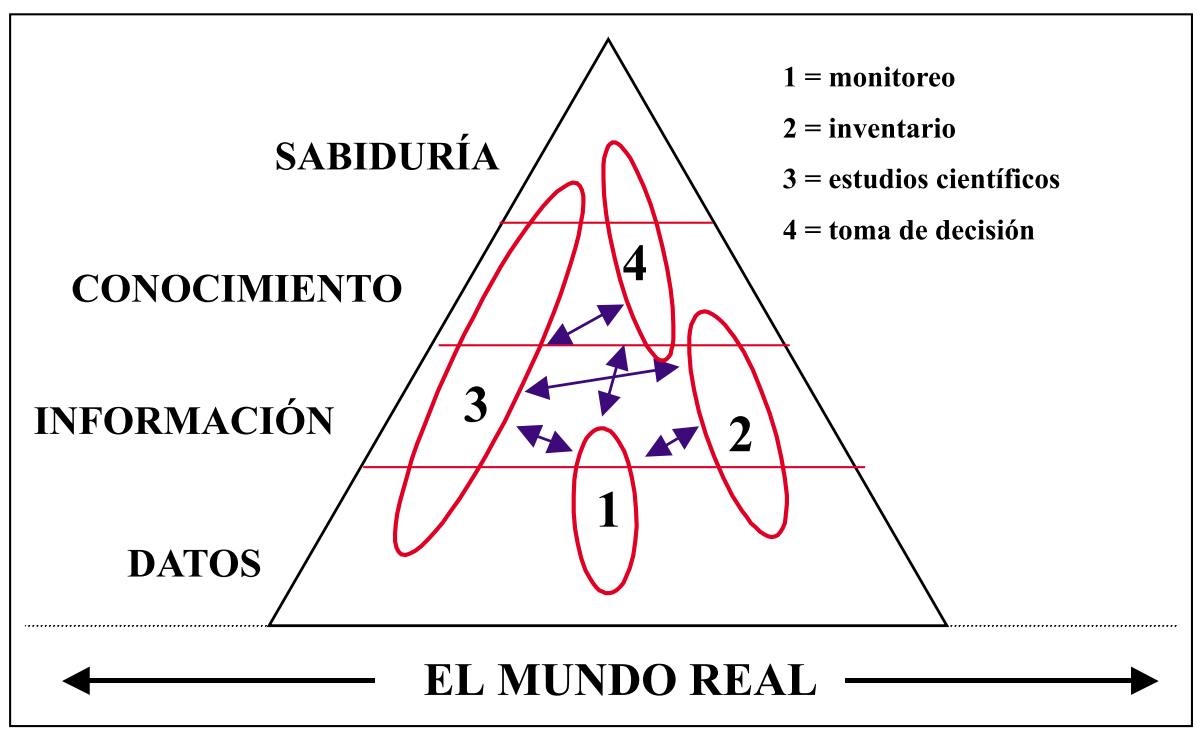

Figura 1. Esquema jerárquico de niveles de información. El mundo real tiene un número infinito de detalles, que se puede entender por la generación de datos (muestras) que, por su parte, pueden ser utilizados para generar información, conocimiento y sabiduría. Los números 1-4 muestran el puesto de diferentes actores en este esquema y las flechas indican necesidades de comunicación.

Es necesario aclarar las relaciones entre datos e información, términos que a menudo suelen ser intercambiables. Los datos generalmente se refieren a las observaciones o medidas descriptivas de una entidad particular o proceso. Son observaciones sencillas de los estados del mundo. Éstos se estructuran fácilmente, se capturan con facilidad, a menudo se cuantifican, y se transfieren también con facilidad. El término información, por su parte, se refiere usualmente a los datos que han sido organizados, integrados y, en algunas ocasiones, analizados. Son datos dotados de pertinencia y propósito. La información generalmente requiere una unidad de análisis, un consenso sobre su significado, y así la intermediación humana es indispensable; por tales razones, el conocimiento y la sabiduría pueden ser derivados de la información a través de su análisis adicional, aplicando teorías científicas, interpretación y entendimiento. La sabiduría particularmente también incluye reflexión, síntesis y contexto, siendo a veces difícil de estructurar y capturar.

\section{INFORMACIÓN Y SOCIEDAD}

El flujo de la información entre los diferentes niveles no es automáticamente efectivo, y hay que facilitar el proceso con herramientas adecuadas. Entre los principales problemas se puede considerar aspectos relacionados con la motivación (desinterés para profundizar el conocimiento), acceso (carencia, restricciones en disponibilidad, material no publicado o inadecuado), así como algunos requerimientos de los usuarios (formato, documentación inadecuada, pobre calidad, credibilidad científica limitada). Así mismo, los aspectos financieros y referidos a los derechos intelectuales tienen que ver con la eficacia de la distribución de información en la sociedad.

Aunado a esto, es necesario destacar la tensión experimentada por muchas instituciones al momento de decidir entre la obtención de resultados en un corto plazo y el deseo de acumular información a largo plazo. Hay que resaltar el gran impacto de generar todo un bagaje de información con una orientación definida de largo plazo (Hawksworth, 1995). De este modo, la información resultante sería útil para ser usada por diversos tipos de audiencias y ocasiones, tanto actualmente como en el futuro.

En la sociedad moderna, cada institución tiene su propia cultura de informática, y aquellas entidades que desarrollan sus prácticas sobre la base de una visión y política fija, son actores principales de la llamada sociedad de informática. Estos temas son muy importantes y deben ser considerados como temas prioritarios por las 
instituciones de investigación, enseñanza o administración que deseen ser considerados como referentes importantes en su campo.

Cuando se analiza la situación actual de la información sobre diversidad biológica en el Perú, es posible definir algunos aspectos que puede describir la realidad: poco o ningún acceso, desconocimiento de la realidad, y casi o ninguna sistematización, entre otros. Los datos y la información sobre diversidad biológica amazónica se encuentran dispersos en varios formatos, y en ocasiones no están documentados o sistematizados. Reorientar la tendencia es todo un reto. La mayoría de los actores nacionales en este campo carecen orientación y recursos para adoptar una política fija de informática y así obtener una posición respetada en las redes de información de la "sociedad de la información".

\section{INFORMACIÓN EN DIVERSIDAD BIOLÓGICA}

La descripción y clasificación de las especies vivientes y el mejor entendimiento de los procesos ecológicos y evolutivos que contribuyen a la diversidad biológica pueden considerarse entre los mayores objetivos científicos del nuevo siglo (Hawksworth, 1995; Wilson, 2000). En la ciencia aplicada, el complemento del conocimiento taxonómico y ecológico es necesario para la efectiva práctica de la conservación, para la bioprospección y para estudios de impacto ambiental.

Uno de los aspectos fundamentales de la información sobre diversidad biológica son las bases de datos de las colecciones biológicas, esenciales para unificar los esfuerzos para la creación de una imagen detallada de la biota. Estas colecciones poseen información sobre taxonomía, sistemática, e inclusive de genética, localización geográfica, uso de hábitat, uso tradicional y otros. Además constituye una muestra de que la información referida se sustenta con un ejemplar concreto (Figura 2). Los datos provenientes de observaciones también son importantes, pero en términos de validación no proveen las características de verificación de los ejemplares biológicos.

La conversión de los datos de los ejemplares en información computarizada permite el uso óptimo de los datos. Es necesario tener en cuenta que, a pesar de lo importante y esencial que es manejar la información en bases de datos computarizados, éstas nunca reemplazarán a la información de los ejemplares biológicos per se, los cuales finalmente constituyen la única prueba fehaciente de que los datos encontrados son válidos, proporcionando así, el único medio para comprobar la veracidad de su "identidad" en el futuro.

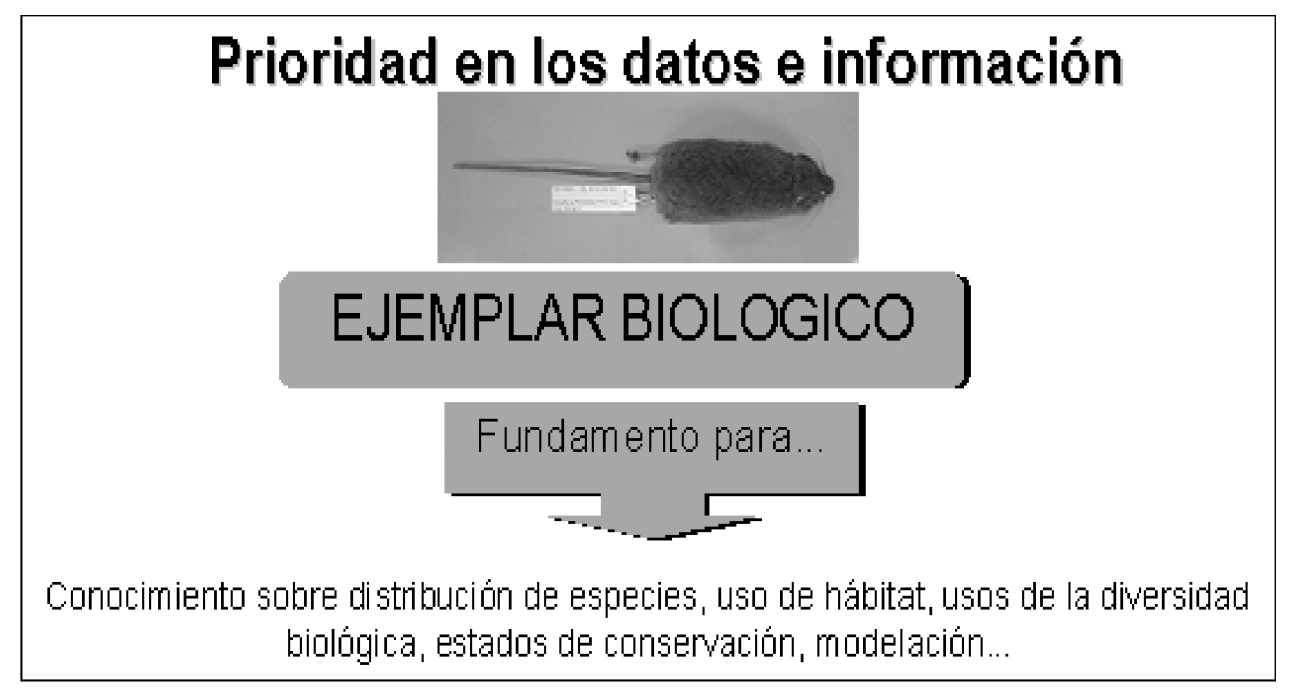

Figura 2. Las colecciones científicas constituyen un valioso banco de datos que compilan información taxonómica, sistemática y ecológica sobre las especies y sus hábitats; por tanto deben estar bien organizadas y sistematizadas. 
En la Amazonía peruana los factores ambientales son muy importantes en su relación con la diversidad biológica, y al mismo tiempo son inadecuadamente conocidos, por lo que su inclusión en un sistema de información es indispensable (BIODAMAZ, 2001a). El Instituto de Investigaciones de la Amazonía Peruana - IIAP, aporta a SIAMAZONIA con un servidor de mapas ambientales que incluye, por ejemplo, un mosaico de las imágenes de satélite de la selva baja de la Amazonía peruana y diversos tipos de mapas temáticos que intentan satisfacer esta necesidad (Figura 3).

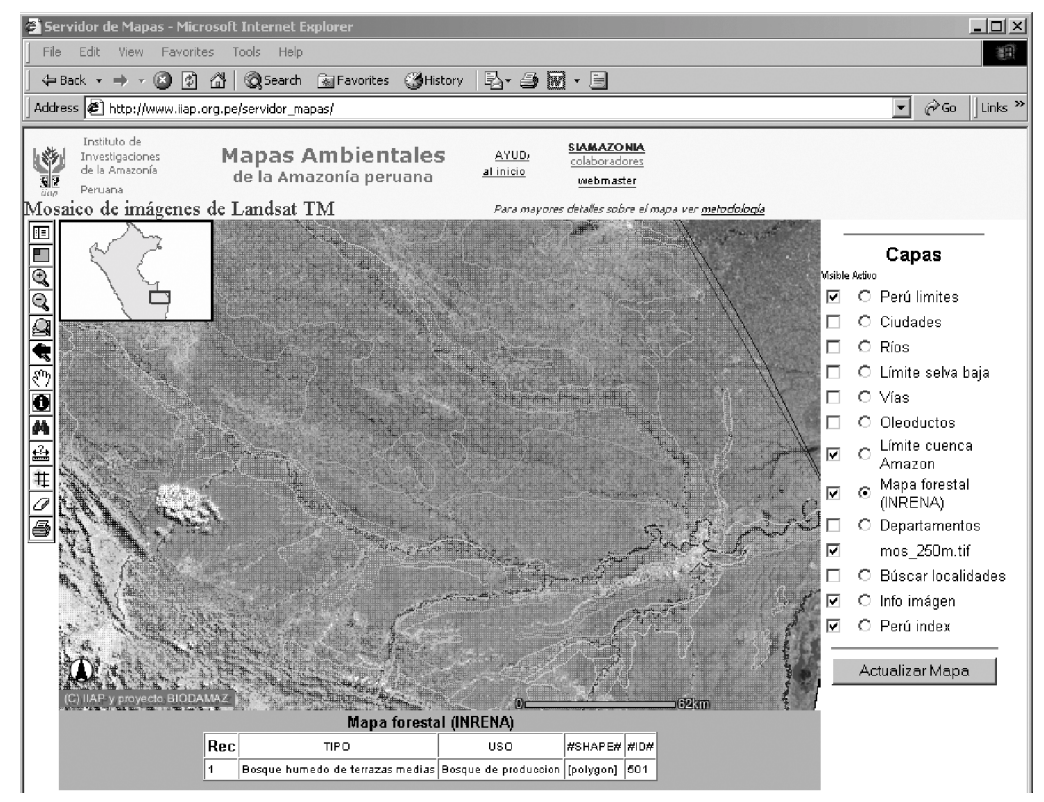

Figura 3. Ejemplo de un servidor de mapas en Internet (proporcionado por el IIAP). En este caso se muestra el departamento de Madre de Dios con un mosaico de las imágenes de satélite juntos, con cobertura semi-transparente del Mapa Forestal del Instituto Nacional de Recursos Naturales (INRENA).

\section{FORMATO DE DATOS}

Los datos numéricos pueden ser datos primarios y datos derivados. Los datos primarios son obtenidos directamente de muchos tipos de observaciones, variando desde conteos de especies en lugares particulares, hasta monitoreo de la precipitación o crecimiento de árboles. Los datos derivados son obtenidos de la manipulación y análisis de otros grupos de datos numéricos. Por ejemplo, la información sobre temperatura, precipitación y altitud de un lugar particular (todos son datos numéricos), pueden ser usados para predecir las zonas de vida según la clasificación de Holdridge.

Es necesario definir un formato para el registro de los datos computarizados en las bases de datos. Por ejemplo, los datos categóricos son clasificados o codificados con datos numéricos, los cuales podrían incluir registros de variables tales como tipo de suelo, tipo de bosque, especies, y designación de áreas protegidas. Son estructurados usualmente a través de un tesauro diccionario de datos. Por ejemplo, la clasificación de datos sobre percepción remota resulta de la derivación de categorías de datos numéricos.

Los datos espaciales, como mapas e imágenes, han sido siempre una valiosa herramienta para el almacenamiento y presentación de la información, debido a que proporcionan una fácil identificación de la naturaleza del paisaje. Es necesario que todos los datos levantados en el campo sean georeferenciados y manejados en un contexto de estándares que permitan su posterior uso en un Sistema de Información Geográfica (SIG). Básicamente, cualquier característica que puede ser georeferenciada puede ser localizada en un mapa, tal como localización de especies, tipos de vegetación, factores ambientales, y otros. 


\section{HERRAMIENTAS DE INFORMÁTICA}

Los diferentes actores que forman parte de las redes de información y comunicación sobre la diversidad biológica y ambiental incluyen los productores y poseedores de información, los expertos de informática, el sector educativo en todos sus niveles, los planificadores que transforman y proporcionan los datos a los tomadores de decisiones, y los políticos que la usan para definir prioridades e iniciar proyectos. Para facilitar los propósitos y satisfacer las necesidades de cada uno de ellos, el uso de las herramientas de informática debe ser orientado en tal forma que se integre óptimamente al interés y las posibilidades de cada actor. El propósito de SIAMAZONIA es servir a todos estos usuarios.

A partir del proveedor de conocimientos, la mayor parte de los datos se puede dominar utilizando los manejadores de bases de datos (DBMS, Database Management System). Estos incluyen sistemas de manejo de bases de datos relacionales (RDBMS), lenguaje estructurado de consultas (SQL, Standard Query Language), y sistema de bases de datos distribuidas; esta última permite acceso a usuarios con amplias necesidades. En las bases de datos distribuidas pueden surgir problemas debido a la diferente tecnología en cada lugar. Sin embargo, se esta desarrollando una posible solución: "sistema de manejo de bases de datos federado", el cual permite comunicación entre DBMSs de diferentes modelos de datos, marca y métodos de acceso. Al mismo tiempo, cada DBMS local permanece autónomo.

Ante la diversidad de formas de presentar la información, la estandarización surge como una alternativa en la cual la informática ofrece muchas ventajas, sobre todo en lo que se refiere a intercambio de información. Actualmente, en algunas instancias de investigación se han iniciado trabajos para contar con sistemas de información compartidos, por ejemplo para estudios forestales y de silvicultura (Rose y Ugalde, 1988). La armonización, fundamentalmente, necesita la definición de campos y tipos de contenido de información para cada muestra o registro. Una vez concordado y establecido un mínimo de datos, las consultas a la información ejecutadas por los nodos que participan en este ejercicio pueden combinar varias bases de datos, de manera que el resultado sea el más integrado posible.

En lo referente a la información espacial, sería indispensable que todos los registros presenten su contexto espacial tan precisamente como sea posible. En la mayoría de casos esto no es más que las coordenadas geográficas de los puntos referidos, facilitando su inclusión en diversos tipos de análisis utilizando las herramientas de SIG. La amplia accesibilidad de los equipos de posicionamiento automático (GPS, Global Positioning System) hace razonable sugerir que, en todos los futuros estudios de campo, las observaciones se registren junto con las coordenadas geográficas de las localidades respectivas.

Muchos registros de fenómenos biológicos y medioambientales se encuentran en forma descriptiva (narrativa), y sólo puede obtenerse en forma de texto. Es importante el uso de palabras claves, aunque la forma contextual de texto libre puede a menudo ser más informativa. Con el advenimiento de las redes de comunicaciones, se esta dando un renacimiento del uso de texto como forma de almacenamiento, intercambio, recuperación y difusión de información. Las herramientas de red, y servicios de correo electrónico y WWW (World Wide Web), son ideales para la consulta y recuperación de texto. Aún cuando se pueden encontrar diferentes tipos de lenguajes y combinaciones de los mismos para presentar información en el Web, para la mayoría de necesidades, el lenguaje HTML (Hypertext Markup Language) resulta el más apto, pues la información producida de este modo es más sencilla y fácil de utilizar a través del Internet.

El Internet es el medio más accesible, más difundido, de más rápido crecimiento y más económico en la difusión globalizada de información. Si bien es cierto que en el 2002 solo aproximadamente un 5\% de la población en el Perú tiene acceso al Internet ${ }^{5}$, las instituciones de investigación y educativas, públicas y privadas, así como las ONGs, políticos y empresas, en su mayoría tienen acceso a este recurso. Más aun, iniciativas nacionales como la de OSIPTEL $^{6}$ a través de FITEL (Fondo de Inversiones para la Telecomunicación), incrementarán el acceso y uso de este medio en los lugares más remotos del país, incrementando la posibilidad de intercambiar y difundir la información amazónica en nuestro país.

5 Red Científica Peruana (RCP) www.rcp.net.pe

6 Organismo Supervisor de las Telecomunicaciones, Plan Cabinas de Acceso Público a Internet, www.osiptel.gob.pe 


\section{PUBLICIDAD Y AVANCE CIENTÍFICO}

Pública en su naturaleza, la ciencia espera desarrollarse mejor en condiciones de libre acceso a las bases de datos electrónicas u otros tipos de almacén de información (Gardner y Rosenbaum, 1998). La comunidad científica contribuye a construir una perspectiva común de la naturaleza a través de los descubrimientos y las críticas constantes de los logros obtenidos. La política actual de algunas revistas científicas es que, cuando un artículo es publicado, los archivos de datos y sus métodos y resultados deben ser depositados en una base de datos de acceso público. De este modo, el intercambio de ideas, metodologías y datos dentro de la comunidad de investigación constituye una necesidad.

Con respecto a información sobre la diversidad biológica, es apropiado pensar que las bases de datos en general son de libre acceso (dominio público). Así mismo, es indispensable que se ordenen de tal forma que la fuente o custodio de la información esté claramente identificado; de esta manera se garantiza que el origen de la información sea siempre conocido. De igual manera, el acceso de la información no debe estar restringido por razones económicas o por privilegios, por ejemplo de tal forma que sólo personas que trabajan en ciertas organizaciones tendrán acceso a los registros. Siendo pública, la información que se presenta también puede contribuir a dar imagen a sus autores, por lo que debe ser de gran interés para cada organización o individuo velar para que los conocimientos que se presentan sean de alta calidad profesional.

Sin embargo, en algunos casos pueden existir restricciones de propiedad intelectual, siendo posible la restricción en la disponibilidad de ciertos datos, a menudo por un período de tiempo dado. Por ejemplo, muchos investigadores prefieren publicar sus resultados antes que compartir sus datos originales con el resto del mundo. Otro ejemplo de restricciones bien sustentadas es el caso de distribución de especies en peligro de extinción o amenazadas, donde es mejor no presentar información muy precisa sobre las localidades para evitar cualquier intervención en las mismas. Estas y otras reglas específicas deben ser definidas por el proveedor de información y el conjunto de participantes en un proceso compartido de manejo de información.

\section{PRINCIPIOS BÁSICOS EN SIAMAZONIA}

El diseño de la estructura de SIAMAZONIA ha sido concebido como una organización de poseedores y generadores de información sobre diversidad biológica. El sistema se enfoca preliminarmente en especies y ecosistemas, incluyendo información a nivel de metadatos, bases de datos de tipo curatorial, taxonómico, ecológico, cartográfico, bibliográfico, y otros aspectos relacionados con las especies y los ecosistemas. En tal sentido, SIAMAZONIA es una red de poseedores de conocimiento en dichos temas.

SIAMAZONIA esta organizado a través de una red nodal, donde cada nodo es la institución o individuo que genera información sobre diversidad biológica de la Amazonía peruana. En esta red nodal se encuentran cuatro niveles de nodos: nodo facilitador, nodos principales, nodos adicionales y nodos de especialistas. La coordinación y funciones secretariales del sistema están a cargo del nodo facilitador (IIAP). Los nodos principales son las instituciones que presentan información de la diversidad biológica bajo las normas definidas en el documento técnico del sistema ${ }^{7}$. Los nodos adicionales son instituciones y organizaciones nacionales e internacionales con conocimientos valiosos de la diversidad biológica amazónica, pero que no cumplen los requisitos definidos para los nodos principales. También se tiene en cuenta la participación de especialistas, definidos como científicos o investigadores individuales con conocimiento de algún campo de la diversidad biológica amazónica del Perú.

El sistema pretende facilitar el intercambio y la divulgación de la extensa y creciente información sobre diversidad biológica almacenada y dispersa en los distintos centros de conocimiento (Figura 4). SIAMAZONIA usa como medio principal un sitio $\mathrm{Web}^{8}$, presentando a los usuarios una interfase común e integrada; sin embargo, los diferentes servicios pueden ser producidos por diferentes nodos (Figura 5). De esta manera, el conocimiento quedará en su lugar de origen y el sistema simplemente refleja los conocimientos disponibles en diversas partes.

7 BIODAMAZ. 2001b. Sección 2.2 Red del Sistema.

8 Dirección de SIAMAZONIA: http://www.siamazonia.org.pe 
Por lo tanto, se puede acceder a información de varios nodos sin necesidad de salir de SIAMAZONIA. Lógicamente, los usuarios pueden también acceder a la información contenida en los nodos vía alguna otra ruta.

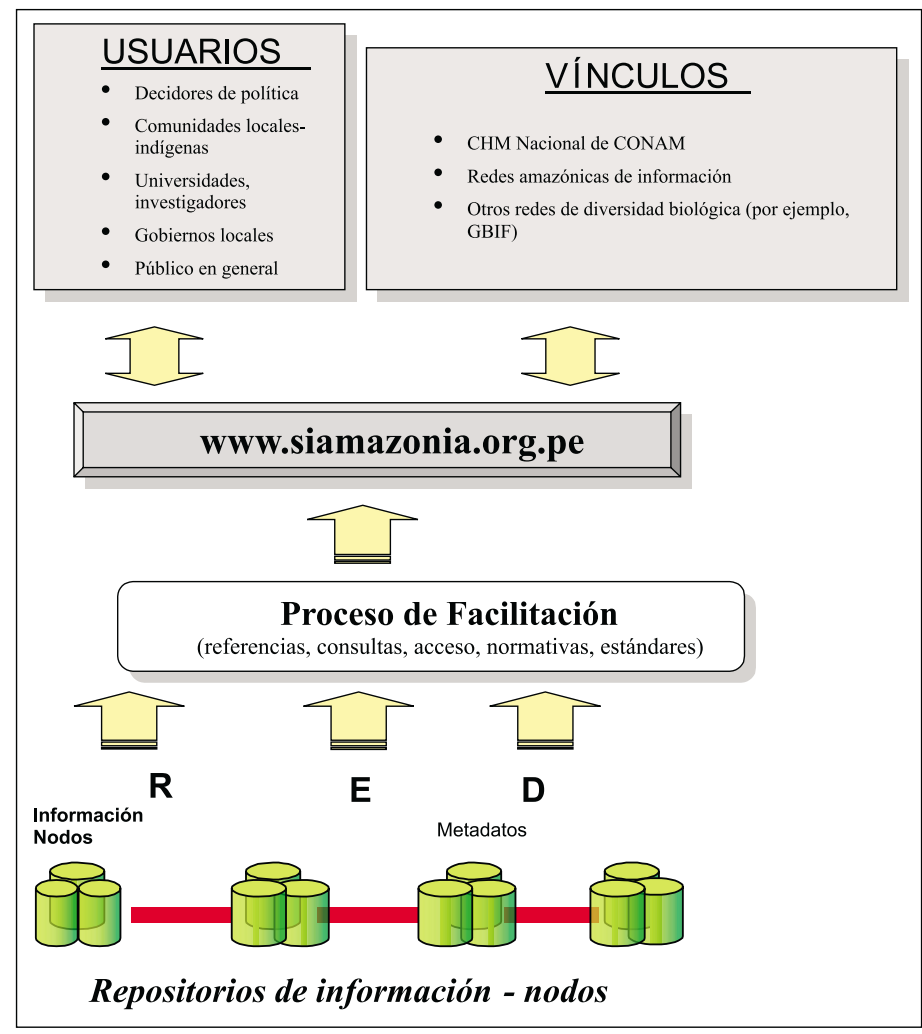

Figura 4. Estructura general de SIAMAZONIA y sus funciones principales.

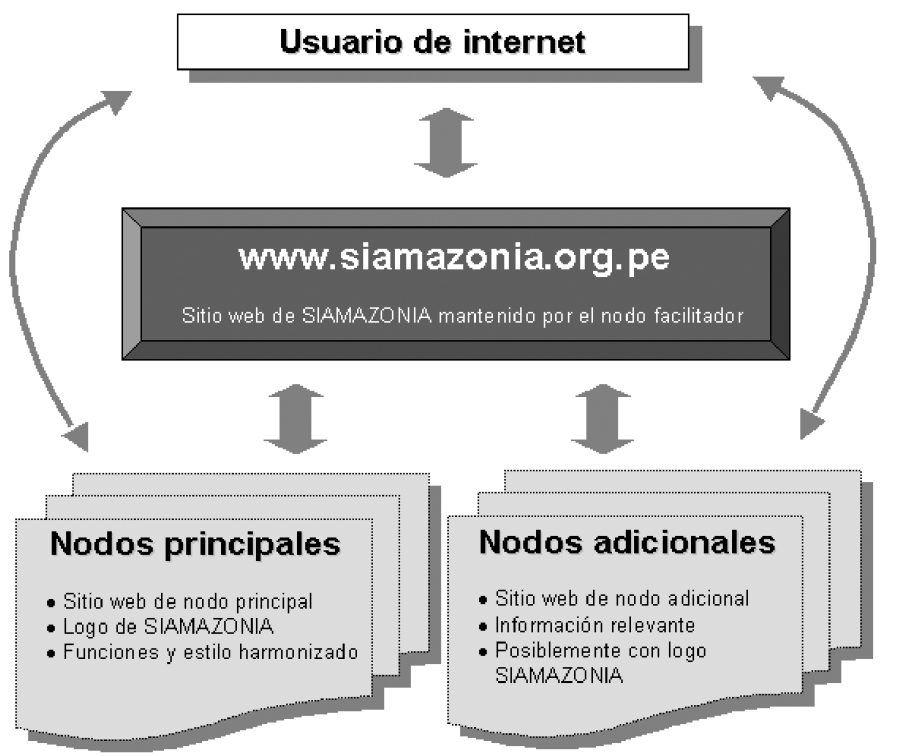

Figura 5. Acceso a los datos producidos y mantenidos por los diferentes nodos de SIAMAZONIA. 
Los nodos adicionales y nodos especialistas que se encuentran fuera del país pueden tener un papel importante en el proceso "repatriación de información". Gran parte de la información relevante sobre la diversidad biológica y las colecciones biológicas se encuentran en países de América del Norte y Europa. Debido a esta situación, es importante poder elaborar mecanismos que nos permitan acceder a esta información desde Perú, y en ciertos casos almacenarla localmente. En este sentido, los poseedores de información en el extranjero pueden actuar como nodos adicionales o especialistas en SIAMAZONIA, presentando información de sus estudios y colecciones de importancia para el Perú, ya sea a través de SIAMAZONIA o en sitios Web propios, que contribuyan al intercambio y difusión de este tipo de información.

\section{EXPERIENCIAS Y PERSPECTIVAS}

El desarrollo de SIAMAZONIA comenzó con una fase de gabinete, con especialistas de la Universidad de Turku y del IIAP en el año 2000 (Figura 6). A inicios del 2001 se presentó la primera propuesta del sistema en cinco talleres de consulta regionales: en Ucayali (Pucallpa), San Martín (Tarapoto), Madre de Dios (Puerto Maldonado), Loreto (Iquitos) y Lima. Los aportes recibidos en estos talleres fueron cuidadosamente analizados y aplicados, de manera que se desarrolló una versión mejorada del diseño. Finalmente, se elaboró el Documento Técnico $N^{\circ}$ 02: Sistema de Información de la Diversidad Biológica y Ambiental de la Amazonía peruana. Serie BIODAMAZ-IIAP (BIODAMAZ 2001b), y se inauguró el sistema y el sitio web el 23 de noviembre del 2001 (Figura 7). Todas estas fases estuvieron a cargo del proyecto BIODAMAZ.

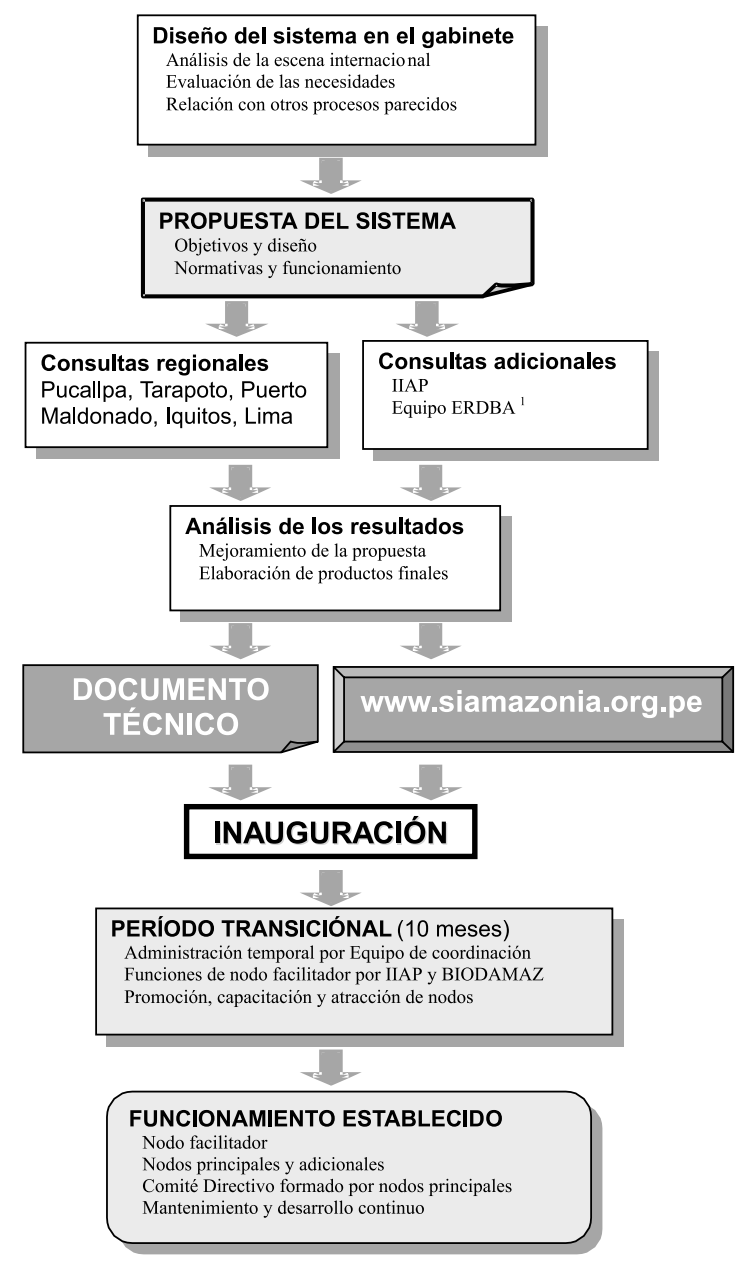

Figura 6. Fases de trabajo en el desarrollo inicial de SIAMAZONIA (2000-2002).

${ }^{1}$ ERDBA: Estrategia Regional de la Diversidad Biológica Amazónica (BIODAMAZ, 2001a). 


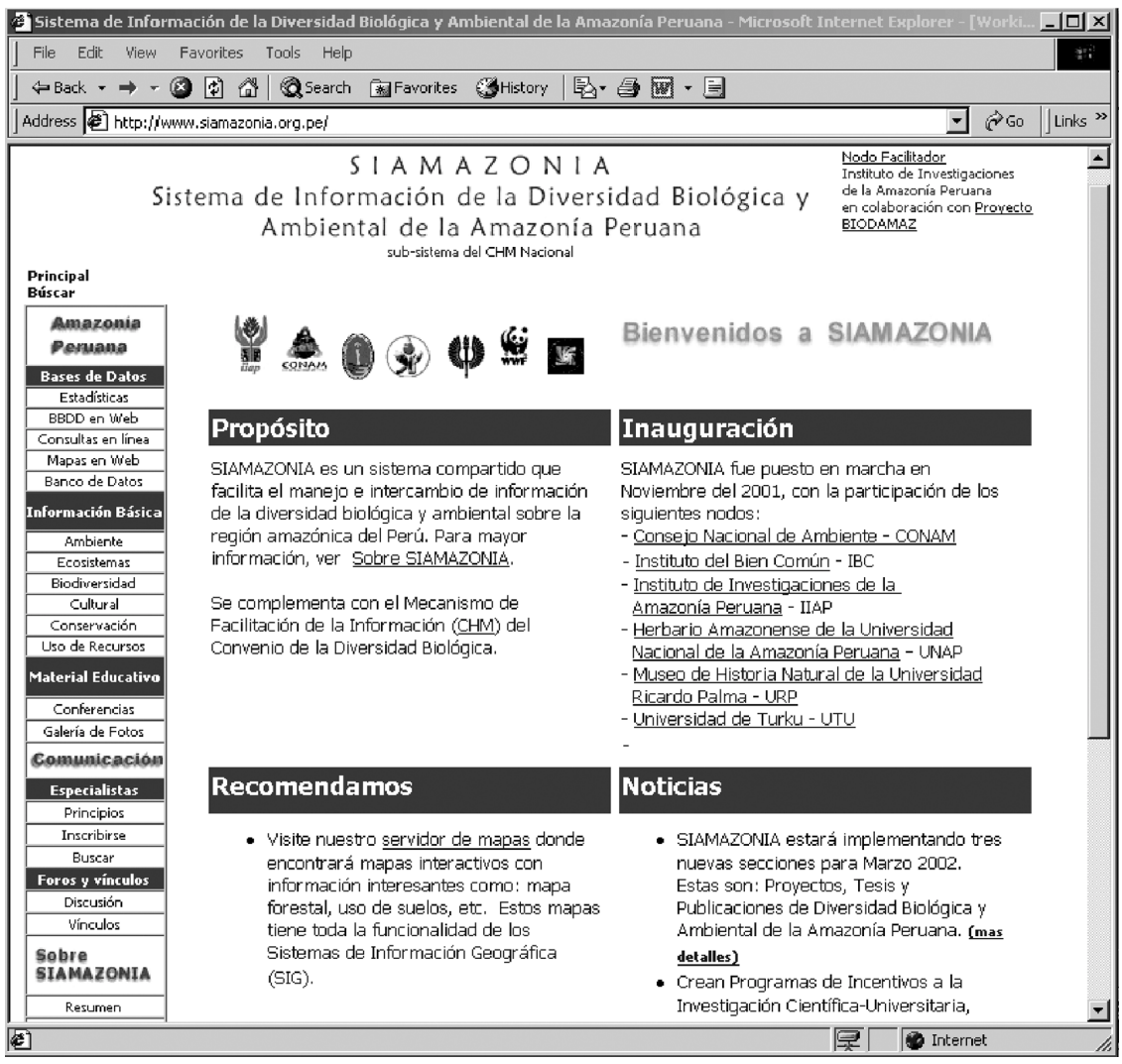

Figura 7. Página inicial de SIAMAZONIA en febrero de 2002.

Después de su inauguración, SIAMAZONIA entró en un período de funcionamiento en transición, donde el IIAP actúa como nodo facilitador. Durante este período de transición no se han consolidado nodos principales, por lo que no se ha podido establecer el Comité Directivo - el cual debe estar conformado por los representantes de los nodos principales del sistema. Sin embargo, existen coordinaciones con representantes del IIAP, BIODAMAZ y las instituciones que participan en SIAMAZONIA desde su inauguración. Las principales actividades durante el período de transición incluyen promoción general del sistema, enriquecimiento y mejoramiento de sus servicios, integración de SIAMAZONIA en otras redes, y atracción de nuevos nodos para el sistema. Un aspecto clave es la motivación de los nodos de manera que los futuros nodos del sistema pueden apreciar el valor de su participación y sus aportes al conocimiento de la diversidad biológica.

Se considera que las nuevas tecnologías de la información están desarrollándose rápidamente para beneficio de sistemas de información integrada y compartida. Cada nueva generación de las tecnologías de información y comunicación nos presenta nuevos métodos para colectar, manejar e intercambiar información con más eficiencia y eficacia. También las tecnologías de comunicación móvil y aquéllas de posicionamiento automático parecen ofrecer opciones interesantes en el futuro (Burnett y Kalliola, 2000). Las redes de computadoras pueden fácilmente vincular fuentes de información que se encuentran físicamente lejos entre sí dentro de un país o región, y hasta en diferentes continentes. Por lo tanto, es indispensable seguir el avance tecnológico continuamente y adoptar las nuevas herramientas a SIAMAZONIA cuando sea apropiado.

Definitivamente, SIAMAZONIA constituye una primera iniciativa, la cual no sólo debe fortalecer los esfuerzos nacionales sino también que debe marcar la pauta para desarrollar iniciativas en escalas mayores, por ejemplo a nivel de la cuenca amazónica. Todo esto será posible a través de una coordinación conjunta de todos los involucrados, lo cual finalmente hará de SIAMAZONIA una herramienta de trabajo que tiene como base la diversidad biológica y la informática, y que finalmente estamos seguros contribuirá decisivamente al desarrollo 
de toda la sociedad. Esperamos que un aumento significativo en la calidad del manejo de la información amazónica también pueda favorecer la captación de inversiones que promuevan tanto la investigación como el desarrollo de la región.

\section{AGRADECIMIENTOS}

Muchas personas e individuos han participado en el desarrollo de las ideas presentadas en este artículo. Particularmente, queremos agradecer a Hernán Tello Fernández, Sanna Juvonen y Jean Mattos, por su contribución valiosa en este sentido, y a todos nuestros colegas y amigos con quien hemos conversado estos temas en BIODAMAZ y en el IIAP. Así mismo, quisiéramos agradecer profundamente a todos los participantes en los talleres regionales realizados en Ucayali, San Martín, Madre de Dios, Loreto y Lima. Nuestro agradecimiento al proyecto BIODAMAZ, Perú - Finlandia, por la oportunidad de realizar este trabajo.

\section{BIBLIOGRAFÍA}

ARRIAGA, L; CAREAGA, S. 1999. La Red Mexicana de Información sobre Diversidad Biológica. Biodiversitas $N^{\circ}$ 26. Septiembre.

BIODAMAZ. 2001a. Estrategia Regional de la Diversidad Biológica Amazónica. Documento Técnico No 01. Serie BIODAMAZ-IIAP. Iquitos, Perú. 75 pp. (Versión digital disponible en SIAMAZONIA http:// www.siamazonia.org.pe).

BIODAMAZ. 2001b. Sistema de Información de la Diversidad Biológica y Ambiental de la Amazonía Peruana. Documento Técnico $\mathrm{N}^{\circ}$ 02. Serie BIODAMAZ-IIAP. Iquitos, Perú. 55 pp. (Versión digital disponible en SIAMAZONIA http://www.siamazonia.org.pe).

BURNETT, C.; KALLIOLA, R. 2000. Maps in the Information Society. Fennia 178: 1, 81-96.

DOUROJEANNI, M.J. 1990. Amazonía - ¿Que hacer? Centro de Estudios Teológicos de la Amazonía (CETA), Iquitos, Perú. 444 pp.

ENDB. 2001. Perú: Estrategia Nacional sobre la Diversidad Biológica. CONAM. Lima, Perú.

GARDNER, W.; ROSENBAUM, J. 1998. Database Protection and Access to Information. Science 281: 786787.

HAWKSWORTH, D. L. 1995. The resource base for biodiversity assessments. En: Heywood, V.H.; Watson, R.T. (eds.). Global Biodiversity Assessment. Cambridge University Press, UK. 548-605 pp.

KALLIOLA, R.; PUHAKKA, M.; DANJOY, W. (eds.). 1993. Amazonía Peruana. Vegetación Húmeda Tropical en el Llano Subandino. PAUT-ONERN. Jyväskylä, Finlandia. 265 pp.

LAIHONEN, P.; RÖNKÄ, M.; TOLVANEN, H.; KALLIOLA, R. 2003. Geospatially structured biodiversity information as a component of a regional biodiversity clearing house. Biodiversity and Conservation 12:103-120.

ROSE, W.; UGALDE, L. 1988. Database management applications in forestry research. International Workshop on Data Base Management Applications in Forestry Research. Turrialba (Costa Rica). 20-25 Junio. Serie Técnica. Informe Técnico (CATIE). no. 143, 102 pp.

SAARENMAA, H. 1998. The Global Biodiversity Information Facility: Architectural and Implementation Issues. European Environment Agency, Copenhagen. Technical Reports 34. 34 pp. http:// www.eionet.eu.int/gbif/gbif-implementation-latest.html.

WILSON, E. 2000. A Global Biodiversity Map. Science 289 (5488): 2279. 